The black dotted line: Controls without BP $(r=0.69, p<0.0001)$. The solid line: Preterm AGA without BP $(r=0.38, p=0.11)$, the red dotted: preterms AGA with $B P(r=0.06, p=N S)$.

At 9 years, preterm AGA with BP $(n=13)$ had lower length SDS $(p=0.003)$, weight SDS $(p=0.006)$ and head circumference SDS and a tendency to lower height catch-up $(p=0.09)$ compared to preterm AGA without BP $(n=18)$. Fasting levels of IGF-I, insulin and leptin were lower in all Preterms with BP.

Preterms with SP $(n=8)$ had a lower height catch-up $(\mathrm{p}=0.009)$ compared to those without SP $(\mathrm{n}=30)$.

Conclusion Children born preterm have an increased risk for SP and BP. These disorders are associated with reduced catch up in height.

\section{REPEAT COURSES OF ANTENATAL CORTICOSTEROIDS FOR PRETERM BIRTH AND RISK FOR METABOLIC SYNDROME IN YOUNG ADULTHOOD}

doi:10.1136/archdischild-2012-302724.0453

${ }^{1} \mathrm{H}$ Norberg, ${ }^{2} \mathrm{~J}$ Stålnacke, ${ }^{2} \mathrm{R}$ Diaz Heijtz, ${ }^{3} \mathrm{AC}$ Smedler, ${ }^{2} \mathrm{H}$ Forssberg, ${ }^{1} \mathrm{M}$ Norman. ${ }^{1}$ Department of Clinical Science, Intervention and Technology; ${ }^{2}$ Department of Women's and Children's Health, Karolinska Institutet; ${ }^{3}$ Department of Psychology, Stockholm University, Stockholm, Sweden

Background Preterm birth is associated with later hypertension and diabetes. One explanation for this association could be that exposure to antenatal corticosteroids (ACS), especially if repeated, induce adverse long-term effects. There are no data on whether repeat courses of ACS are associated with health problems later in life. The aim of this study was to assess whether repeat courses of ACS correlate to metabolic syndrome later in life.

Methods In a population-based cohort we measured BMI, blood pressure, arterial stiffness, blood lipids and glucose tolerance in 58 subjects ( 36 boys, age 14 to 26 years) exposed to 2-9 weekly courses of antenatal betamethasone. Subjects exposed to a single course ( $n=25,14$ boys) and unexposed subjects ( $n=44,25$ boys) were included as comparison groups.

Results As compared to unexposed controls, subjects exposed to repeat courses of ACS did not differ in BMI (mean difference $0.6 \mathrm{~kg} /$ $\mathrm{m}^{2}, \mathrm{p}=0.5$ ), mean systolic or diastolic blood pressure (mean diff $1 \mathrm{mmHg}, \mathrm{p}=0.78-0.83$ ), arterial stiffness assessed by pulse wave analysis (mean diff $0.1 \%, \mathrm{p}=0.50$ ), triglyceride (mean diff $0.1 \mathrm{mmol} / \mathrm{L}$ ), total cholesterol (mean diff $0 \mathrm{mmol} / \mathrm{L}$ ), LDL/HDL ratio (mean diff 0.1), Lipoprotein(a) (mean diff. $61 \mathrm{mg} / \mathrm{L}$ ), ApolipoproteinB/ApolipoproteinA1 ratio (mean diff 0.01$),(p=0.33-0.91)$ or glucose tolerance assessed by HOMA-index (mean diff $0, \mathrm{p}=0.84$ ). Subjects exposed to a single course of ACS did not differ from the other groups in any of the variables above.

Conclusions Repeat courses of ACS do not correlate to metabolic syndrome in young adulthood. This observation has clinical implications for the ongoing discussion about safety of antenatal steroids.

\section{HEPATIC GLYCOGENOSIS IN TYPE I DIABETES MELLITUS: REPORT OF TWO CASES AND REVIEW OF THE LITERATURE}

doi:10.1136/archdischild-2012-302724.0454

LW de Groot, WB Geven. Pediatrics, Martini Hospital, Groningen, The Netherlands

Aim Hepatic glycogenosis is an underrecognized cause of serum transaminase elevations in poorly controlled type I diabetes mellitus, which has a relatively benign course with appropriate treatment. ${ }^{1,2}$ Objective of this study is to describe the aetiology, clinical presenting symptoms and treatment options.

Methods A report of two adolescents with poor controlled diabetes mellitus, hepatomegaly and serum transaminase elevations and a literature review.
Results Both cases presented with abdominal pain and hepatomegaly, combined with nausea and dyspeptic complaints. Laboratory investigation revealed marked elevation of serum transaminase levels. Synthetic function of the liver stayed intact. Abdominal ultrasound showed isolated, homogenous hepatomegaly, without other abdominal abnormalities. In one case liver biopsy was performed, showing hepatic glycogenosis. Other causes for hepatomegaly were excluded. With improved diabetic control all complaints improved within three weeks, with normalisation of serum transaminase levels.

Review of literature that hepatic glycogenosis, not frequently described, is an important complication of type I diabetes mellitus. Hepatic glycogenosis as result of glycogen storage in hepatocytes, caused by periods of hyperglycaemia and frequent insulin boluses. This process is reversible with improved glycaemic control. ${ }^{1,2}$

Conclusions Hepatic glycogenosis is a important complication of type I diabetes mellitus which can be reversible with the proper treatment. Therefore, medical attention is necessary.

\section{References}

1. C.F.J. Munns et al. Hepatic glycogenosis: Reversible hepatomegaly in type 1 diabetes. Journal of Paediatrics and Child Health 2000; 36:449-452.

2. M. Torbenson et al. Glycogenic Hepatopathy, an underrecognized hepatic complication of diabetes mellitus. The American Journal of Surgical Pathology 2006; 30:508-513.

\section{IMPROVEMENT OF SERUM TESTOSTERONE IN DIABETIC RATS TREATED WITH METFORMIN AND NIGELLA SATIVA}

doi:10.1136/archdischild-2012-302724.0455

${ }^{1} \mathrm{~A}$ Abd ElShaheed, ${ }^{2} \mathrm{KA}$ El-Shamy, ${ }^{2} \mathrm{TH}$ Mekhael, ${ }^{3} \mathrm{~F}$ Adly, ${ }^{4} \mathrm{R}$ Boulos, ${ }^{4} \mathrm{SE}$ Ibrahim, ${ }^{2} \mathrm{NN}$ Fadl. ' $\mathrm{C}$ Child Health; ${ }^{2}$ Physiology; ${ }^{3}$ Pathology, National Research Centre; 'Zoology Department, Faculty of Science, Cairo University, Cairo, Egypt

Background and Aims To evaluate the effect of metformin and Nigella sativa (alone or in combination) on improving the diabetic state of rats.

Methods Male Sprague-Dawley rats weighing 180-200g had induced diabetes using alloxan $(150 \mathrm{mg} / \mathrm{kg})$, then diabetic rats were treated daily for 45 days with metformin $(0.5 \mathrm{~g} / \mathrm{Kg}$.b.wt), Nigella sativa $(1 \mathrm{~g} / \mathrm{Kg} . b . w t)$ or a mixture of metformin + Nigella sativa $(0.25 \mathrm{~g}+1 \mathrm{~g} / \mathrm{Kg}$.b.wt $)$ in a separated three groups and compared with a group of alloxanated diabetic rats as control. HbA1c, serum glucose, lipid profile, microalbuminuria (MA), ALT, AST, insulin, SHBG and total testosterone were measured using ELISA \& spectrophotometer techniques, testis and liver tissue were examined histopathologically.

Results Both metformin and Nigella sativa were comparable in reducing serum glucose of the diabetic rats, furthermore, Nigella sativa showed a hypolipidaemic effect and it also improved liver functions. The level of serum insulin was significantly increased $(\mathrm{P}<0.05)$ in three groups. Importantly, using the mixture of metformin and N.sativa was less effective in improving diabetic state than using metformin or N.sativa alone, although it had improved serum level of testosterone and normalized the structure of testis.

Conclusion Using either metformin or Nigella sativa alone was more effective in improving the diabetic state of rats than using them in combination, although this combination was more effective in improving both serum level of testosterone and the structure of testis. This raise basic questions about the effect of interactions that may occur on using this mixture in the treatment of diabetes that necessitate further studies.

456 SEGMENTAL ACUTE SPINAL CORD SYNDROME: "AS A BOMB" 


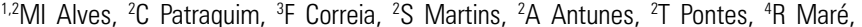
${ }^{2.5 \mathrm{H}}$ Antunes. 'Serviço de Pediatria, Centro Hospitalar São João, Porto; ' $U n i d a d e$ Adolescentes, Serviço de Pediatria, Hospital de Braga, Braga; ${ }^{3}$ Serviço de Pediatria, Centro Hospitalar Alto Ave, Guimarães; ${ }^{4}$ Serviço de Neurologia, Hospital de Braga, Braga; ${ }^{5}$ nstituto de Ciências da Vida e da Saúde (ICVS), Escola de Ciências da Saúde da Universidade do Minho, Laboratório Associado ICVS/3B's, Braga/Guimarães, Portugal

Background The segmental spinal cord syndrome is defined clinically as a neurological injury (incomplete or complete) sensory, motor and autonomic.

Results 13 year old female, previously healthy with a sudden onset of severe pain in the inferior limbs, with loss of strength and sensitivity. No history of fever, recent trauma, infection, vaccination or similar episode. The physical examination revealed areflexic flaccid paraplegia, abolished postural sensitivities, painful hypoesthesia with sensitive level at T8, bilateral indifferent cutaneous plantar reflex response and vesical globe.

A brain and spinal cord magnetic ressonance imaging (MRI) study showed a discreet hyperintensity of the conus, and cerebrospinal fluid analysis was normal. She was hospitalized admitting a possible diagnosis of transverse myelitis and medicated with methylprednisolone intravenously. Due to the absence of clinical improvement, at day five MRI was repeated showing extensive hyperintensity of the conus with restricted diffusion, which extended to the level of T10, diagnostic hypothesis of acute transverse myelitis or acute ischemic injury was made. An etiological study was conducted presenting heterozygosity for Factor V Leiden. On day 32, after submission to physiotherapy, with slight improvement of proximal weakness in the lower limbs, a new MRI showed spinal cord atrophy.

Conclusions In this case, the main challenge is to establish the diagnosis and its etiology. Clinically, transverse myelitis is the most probable diagnosis although by imaging, one cannot exclude spinal cord infarction. Regardless of this issue, the overall prognosis, conditioned by severe initial dysfunction and no recovery in the first 24 hours, is unfavorable.

\section{SELF-ASSESSMENT OF APPEARANCE AND USING OF SLIMIMING SUPPLEMENTS AND ERGOGENIC AIDS AMONG POLISH STUDENTS FROM JUNIOR HIGH SCHOOLS}

doi:10.1136/archdischild-2012-302724.0457

'E Kolarzyk, ${ }^{2} \mathrm{~J} J$ Jośko, ${ }^{2} \mathrm{~J}$ Kasperczyk, 'A Ostachowska-Gąsior. 'Department of Hygiene and Dietetics, Jagiellonian University, Medical College, Krakow; ${ }^{2}$ Department of Environmental Medicine and Epidemiology, Medical University of Silesia, Zabrze, Poland

Dynamic physical and psychological changes connected with adolescence are associated with focusing on appearance. The difference between the imagine figure and real appearance can become a source of frustration and dissatisfaction.

The aim Assessment of body weight acceptance and different actions aiming at appearance improvement among Polish students from junior high schools.

Methods The examinations included 986 students from junior high schools (551girls and 435 boys) at the mean age 13 years and BMI 19.28 \pm 2.85 (girls) and 19.90 \pm 4.51 (boys). An anonymous standardized questionnaire concerning nutritional behaviors was used. Results Dissatisfaction with body mass and general appearance was declared by $31.4 \%$ boys and $40.6 \%$ girls. The majority of these girls used different weight-loss diets (reduction, low-carbohydrate, protein, yoghurt and fruit diets) and nonconventional diets such $\mathrm{dr}$ Atkins' and South Beach. Supplements which stimulate fat tissue burn, metabolic process and thermogenesis and such products as Alli, Bio CLA, Linea and Therm Line II were the most popular. The boys declared taking the ergogenic aids (vitamin and mineral preparations) and supplementation with protein or carbohydrate. The reduction of body mass was the goal for $8 \%$ of boys and they also decided to start a weight-loss diet.
Conclusions Low level of self-assessment and lack of appearance acceptance may lead to disturbances with physical and mental health.

In Poland dietary supplements and nutrients are sold without prescription even in filling stations and supermarkets and we afraid that young people will abuse them to create their figure according to their imagine.

\section{THE WAY OF NUTRITION OF POLISH JUNIOR HIGH SCHOOLS PUPILS}

doi:10.1136/archdischild-2012-302724.0458

'E Kolarzyk, ${ }^{2} \mathrm{~J}$ Jośko, 'A Ostachowska-Gąsior, ${ }^{3} \mathrm{~J}$ Kasperczyk. 'Department of Hygiene and Dietetics, Jagiellonian University, Medical College, Krakow; ${ }^{2}$ Department of Environmental Medicine and Epidemiology, Medical University of Silesia, Zabrze; ${ }^{3}$ Department of Environmental Medicine and Epidemiology, Medical University of Silesia, Krakow, Poland

The proper nutrition is important at every period of human life but particularly in adolescence.

The aim Estimation of the frequency of regular consumption of particular meals and the characterization of nutritional habits of junior high schools pupils.

Methods The examinations included 986 students from junior high schools ( 435 boys and 551 girls) at the mean age 13 years and BMI $19.28 \pm 2.85 \mathrm{~kg} / \mathrm{m}^{2}$ (girls) and $19.90 \pm 4.51 \mathrm{~kg} / \mathrm{m}^{2}$ (boys). An anonymous standardized questionnaire concerning nutritional behaviors was used in the study.

Results Breakfast was eaten regularly by $71.7 \%$ (67.6\% girls and $76.8 \%$ boys); second breakfast: $36.7 \%$ (38.1\% girls and $34.5 \%$ boys); lunch: 87.8 (84.9\% girls and $91.0 \%$ boys); desert $22.1 \%$ (21.7\% girls and $22.3 \%$ boys) ; supper: $73.9 \%$ (66.5\% girls and $82.8 \%$ boys). Basic meals were more often omitted by girls than by boys. $33.6 \%$ of girls and $29.0 \%$ of boys eat something among the main meals. It should be pointed out that $25 \%$ of these young people eat their meals alone. Nearly half of the examined group eat chips, cakes, sweets like Snikers, Mars etc. and drink cola, sprite, pepsi. When pupils were asked about the frequency of fast food consumption $2 / 3$ of them answered "sometimes". The answer "never" was chosen by $19.1 \%$ of girls and $18.2 \%$ of boys.

Conclusion The examined group of pupils showed many nutritional mistakes and the way of nutrition becomes far from traditional Polish pattern and becomes similar to nutritional model present in USA and West Europe.

\section{FAMILY FACTORS MODIFYING THE EATING DISORDERS OF YOUNG PEOPLE IN POLAND}

doi:10.1136/archdischild-2012-302724.0459

${ }^{1} \mathrm{~J}$ Josko, ${ }^{2} \mathrm{E}$ Kolarzyk, ' J Kasperczyk, 'U Marcinkowska, ' $\mathrm{K}$ Lau, ${ }^{1} \mathrm{M}$ Tyrpień, ${ }^{3} \mathrm{~K}$ Helewski. ${ }^{1}$ Chair and Departement of Medicine and Environmental Epidemiology, Medical University of Silesia, Zabrze; ${ }^{2}$ Department of Hygiene and Dietetics, Jagiellonian University, Medical College, Krakow; ${ }^{3}$ Department of Histology and Embryology, Medical University of Silesia, Zabrze, Poland

Background and Aims The independent predictors of eating disorders model, characteristic of the families whose children suffer from anorexia is to prefer to be "good parents" than marriage ties and close family relationship. In those families the influence of a father on the premorbid and the accompanying disease personality of children is underestimated. The role of fathers is especially important during daughters' adolescence, who need then their full acceptance. The aim of the study: is there a relationship between age and education of parents and behaviors that may precede the incidence of anorexia.

Methods 1050 middle school students (13.8 \pm 0.98 year old) from Poland, have been examined using anonymous standardized 